\title{
FINITE MORPHISMS BETWEEN FANO HYPERSURFACES *
}

\section{INSONG $\mathrm{CHOE}^{\dagger}$}

\begin{abstract}
Let $X$ and $\tilde{X}$ be smooth Fano hypersurfaces in $\mathbb{P}^{n+1}, n \geq 3$. We show that if $\operatorname{deg}(X)<2 \operatorname{deg}(\tilde{X})$ then there are no nontrivial finite morphisms $f: X \rightarrow \tilde{X}$.
\end{abstract}

1. Introduction. For a complex projective manifold $X$, the index of $X$ is defined by the largest positive integer $r$ such that there is a line bundle $L$ on $X$ with $K_{X}=r L$. For the morphisms between Fano manifolds, T. Peternell made the following

Conjecture $([\mathrm{P}])$. Let $X$ and $\tilde{X}$ be Fano manifolds and assume $b_{2}(X)=1$. Suppose there is a surjective holomorphic map $f: X \rightarrow \tilde{X}$. Then index $(X) \leq$ index $(\tilde{X})$.

This is a generalization of the following theorem of Lazarsfeld's and its quadric analogue.

THEOREM 1.1. ([La]) Let $X$ be a compact manifold other than a point and let $f: \mathbb{P}^{n} \rightarrow X$ be a surjective morphism. Then $X \cong \mathbb{P}^{n}$.

THEOREM 1.2. ([CS], [PS]) Let $Q$ be a smooth quadric hypersurface of dimension at least 3, $Y$ a smooth variety and $f: Q \rightarrow Y$ a finite surjective morphism. Then either $f$ is an isomorphism or $Y \cong \mathbb{P}^{n}$.

In this paper, we are concerned with the related question for Fano hypersurfaces. The above conjecture can be rephrased as follows:

Question: Let $X$ and $\tilde{X}$ be smooth Fano hypersurfaces in $\mathbb{P}^{n+1}$ of degree $d, \tilde{d}$ respectively and $f: X \rightarrow \widetilde{X}$ a finite morphism. Is then $\tilde{d} \leq d$ ?

Note that the order is reversed since $K_{X}=\mathcal{O}_{X}(d-n-2)$ and $d \leq n+1$. In view of above two theorems, the answer is Yes if either $d$ or $\tilde{d}$ is less than 3 . For $d, \tilde{d} \geq 3$, the answer is also Yes. We prove the following stronger result.

MAIN THEOREM. Let $X$ and $\tilde{X}$ be smooth Fano hypersurfaces in $\mathbb{P}^{n+1}, n \geq 3$, of degree $d, \tilde{d}$ respectively, $d, \tilde{d} \geq 3$. Suppose that there is a finite morphism $f: X \rightarrow \tilde{X}$. Then either $2 \tilde{d} \leq d$ or $f$ is an isomorphism possibly except for the case $(n=4, d=5$ and $\tilde{d}=3$ ). Also, the degree of $f$ is bounded by some number which is determined by $d$ and n, (see (I) of Lemma 3.1, 3.2 and 3.3 for the explicit expression of the bound).

Obviously, there exist nontrivial finite morphisms $f: X \rightarrow \tilde{X}$ for some pair of hypersurfaces $X$ and $\tilde{X}$, if $\tilde{d}$ divides $d$. In this sense, the conclusion $2 \tilde{d} \leq d$ is sharp. The exceptional case $(n=4, d=5$ and $\tilde{d}=3)$ lies on a sharp boundary for which our computational method cannot be applied. We believe that there are no finite morphisms in this case, but its proof seems to require another line of argument.

For general hypersurfaces, E. Amerik observed the following

TheOREM 1.3. ([A], Corollary 2.2) Let $X$ be a general hypersurface in $\mathbb{P}^{n+1}$ which is not a quadric or a 2-dimensional cubic, $\widetilde{X}$ a smooth projective variety (not a

*Received October 9, 2002; accepted for publication May 28, 2003.

†School of Mathematical Sciences, Seoul National University, Seoul 151-747, Korea (ischoe@math.snu.ac.kr). The author was supported by the Brain Korea 21 Project in 2002 and by KOSEF 98-0701-01-5-L. 
point) and $f: X \rightarrow \widetilde{X}$ a surjective morphism. Then the Hodge numbers of $\widetilde{X}$ coincide either with that of $X$ or with that of $\mathbb{P}^{n}$.

Combined with this, our result has the following

COROLlaRY 1.1. Let $X$ be a general hypersurface in $\mathbb{P}^{n+1}, n \geq 3$, of degree $d \geq 3, f: X \rightarrow \widetilde{X}$ a finite morphism to any hypersurface $\widetilde{X}$ in $\mathbb{P}^{n+1}$, other than $\mathbb{P}^{n}$. Then $f$ is an isomorphism.

Proof. Two hypersurfaces of different degree have different n-th Betti numbers $b_{n}$ except for the pair $\mathbb{P}^{n}$ and the quadric $Q^{n}$ (see [Le], pp.193-194). From above Theorem 1.3, we have $\operatorname{deg}(X)=\operatorname{deg}(\tilde{X})$. If $d \geq n+2$, then by Hurwitz formula

$$
K_{X}=f^{*}\left(K_{\tilde{X}}\right)+R
$$

$f$ is an isomorphism. If $d<n+2, f$ is also an isomorphism by the Main Theorem. (

The main tool for our proof is the Hurwitz-type formula devised by E. Amerik, M. Rovinsky, and A. Van de Ven ([ARV]). Recently A. Beauville used this to prove that a smooth projective hypersurface of dimension greater than 2 admits no endomorphisms of degree greater than 1 ([B]). He applied the Hurwitz-type formula to maps of arbitrarily large degree by using iteration of given endomorphism. Here we apply the same formula with a direct computation to arbitrary finite morphisms.

In section 2, the Hurwitz-type formula is discussed. It will be presented in an explicit form adapted to our case. The section 3 is devoted to the proof of Main Theorem. The main tools is the Hurwitz-type formula , But we take care of some boundary cases by different methods, because the Hurwitz-type formula no longer works for them.

2. Hurwitz-type formula . Let $X$ and $\tilde{X}$ be smooth hypersurfaces in $\mathbb{P}^{n+1}$ of degree $d$ and $\tilde{d}$, respectively. We assume $n \geq 3$. Then $\operatorname{Pic}(X) \cong \operatorname{Pic}(\widetilde{X}) \cong \mathbb{Z}$ and they are generated by the hyperplane section classes $H_{X}$ and $H_{\tilde{X}}$, respectively. Then the following Hurwitz type inequality holds.

LEMma 2.1. ([ARV], $[B])$ Let $f: X \rightarrow \tilde{X}$ be a finite map between hypersurfaces $X$ and $\tilde{X}$ as above, and let $m$ be the number satisfying $f^{*} H_{\tilde{X}}=m H_{X}$. Then

$$
\frac{1}{\tilde{d}} c_{n}\left(\Omega_{\widetilde{X}}^{1}(2)\right) \leq \frac{1}{m^{n} d} c_{n}\left(\Omega_{X}^{1}(2 m)\right)
$$

Proof. Note that $\Omega_{\mathbb{P}^{n+1}}^{1}(2)$ is globally generated, hence so is $\Omega_{\widetilde{X}}^{1}(2)$. By [ARV] Corollary 1.2 ,

$$
\operatorname{deg} \cdot c_{n}\left(\Omega_{\widetilde{X}}^{1}(2)\right) \leq c_{n}\left(\Omega_{X}^{1}(2 m)\right)
$$

The inequality (2.1) follows from $\operatorname{deg} f=m^{n} \frac{H_{X}^{n}}{H_{\tilde{X}}^{n}}=m^{n} \frac{d}{\tilde{d}}$.

To compute both sides in (2.1) explicitly, we use the Euler sequence and the conormal sequence twisted by $2 m H_{X}$ :

$$
\begin{aligned}
& \left.0 \rightarrow \Omega_{\mathbb{P}^{n+1}}^{1}(2 m)\right|_{X} \rightarrow\left((2 m-1) H_{X}\right)^{n+2} \rightarrow 2 m H_{X} \rightarrow 0, \\
& \left.0 \rightarrow(2 m-d) H_{X} \rightarrow \Omega_{\mathbb{P}^{n+1}}^{1}(2 m)\right|_{X} \rightarrow \Omega_{X}^{1}(2 m) \rightarrow 0 .
\end{aligned}
$$


From these we get

$$
c\left(\Omega_{X}^{1}(2 m)\right)=\left(1+(2 m-1) H_{X}\right)^{n+2}\left(1+2 m H_{X}\right)^{-1}\left(1+(2 m-d) H_{X}\right)^{-1} .
$$

By using residue theorem, for $d \neq 2 m$ we get

$$
c_{n}\left(\Omega_{X}^{1}(2 m)\right)=\frac{2 m(d-1)^{n+2}-d(2 m-1)^{n+2}+(-1)^{n}(d-2 m)}{2 m(d-2 m)} .
$$

In particular,

$$
c_{n}\left(\Omega_{X}^{1}(2)\right)=\frac{2(d-1)^{n+2}-d+(-1)^{n}(d-2)}{2(d-2)} .
$$

For $d=2 m$, we have

$$
c_{n}\left(\Omega_{X}^{1}(2 m)\right)=\frac{(d n+d+1)(d-1)^{n+1}+(-1)^{n}}{d} .
$$

For convenience, we will use the following bounds:

Lemma 2.2. For any hypersurface $X$ in $\mathbb{P}^{n+1}$ of degree $d$ and for a positive integer $m$, we have

$$
c_{n}\left(\Omega_{X}^{1}(2 m)\right)<\left\{\begin{array}{clc}
\frac{d(2 m-1)^{n+2}}{2 m(2 m-d)} & \text { for } \quad d<2 m \\
(n+2)(d-1)^{n+1} & \text { for } \quad d=2 m \\
\frac{(d-1)^{n+2}}{d-2 m} & \text { for } \quad d>2 m
\end{array}\right.
$$

and

$$
c_{n}\left(\Omega_{X}^{1}(2)\right)>d(d-1)^{n}
$$

Proof. (2.5) is direct from (2.2) and (2.4). Since $(d-1)^{2}=d(d-2)+1$, we get the bound (2.6) from (2.3).

3. Proof of Main Theorem. The basic strategy of proof is the repeated application of the Hurwitz-type inequality (2.1). Frequently we will invoke the bounds (2.5) and (2.6). Throughout this section we work under the following Situation $(*)$ :

- $X$ and $\tilde{X}$ are smooth Fano hypersurfaces in $\mathbb{P}^{n+1}(n \geq 3)$ of degree $d$ and $\tilde{d}$ respectively $(3 \leq d, \tilde{d} \leq n+1)$.

- $f: X \rightarrow \widetilde{X}$ is a finite morphism.

- $H_{X}$ and $H_{\tilde{X}}$ are the hyperplane section classes generating $\operatorname{Pic}(X), \operatorname{Pic}(\tilde{X})$ respectively.

- $m$ is the number satisfying $f^{*} H_{\tilde{X}}=m H_{X}$, and so $\operatorname{deg}(f)=m^{n} d / \tilde{d}$. From (2.1) and (2.6), we get

$$
(\tilde{d}-1)^{n}<\frac{1}{m^{n} d} c_{n}\left(\Omega_{X}^{1}(2 m)\right),
$$

where the righthand side has upper bound given by the inequality (2.5). 
We divide the degree range into several cases and investigate each of them. First we prove

LEMMA 3.1. Under the situation (*), assume $\tilde{d} \geq 5$. Then

(I) $m<\frac{2(d-1)}{\tilde{d}-1}$,

(II) if $d<2 \tilde{d}-1$, then $f$ is an isomorphism (hence $d=\tilde{d}$ ) and (III) if $d=2 \tilde{d}-1$, then $m=2$.

Proof of (I). (i) First suppose $m \geq d$. Then from (2.5) and (3.1),

$$
4^{n}<\frac{1}{m^{n} d} \cdot \frac{d(2 m-1)^{n+2}}{2 m(2 m-d)}<\frac{1}{m^{n}} \cdot \frac{(2 m)^{n+2}}{2 m^{2}}=2^{n+1},
$$

which is impossible.

(ii) Next if $m<d<2 m$, then

$$
4^{n}<\frac{1}{m^{n} d} \cdot \frac{d(2 m-1)^{n+2}}{2 m}<m \cdot 2^{n+1} \leq(d-1) \cdot 2^{n+1} \leq n \cdot 2^{n+1} .
$$

This is also impossible.

(iii) If $d=2 m$, then

$$
4^{n}<\frac{1}{m^{n} d} \cdot(n+2)(d-1)^{n+1}<\frac{(n+2)(2 m)^{n+1}}{2 m^{n+1}}=(n+2) \cdot 2^{n},
$$

which is impossible.

From (i), (ii) and (iii), we get $2 m<d$.

Now suppose $m \geq \frac{2(d-1)}{\tilde{d}-1}$. Then from (2.5) and (3.1) again,

$$
(\tilde{d}-1)^{n}<\frac{1}{m^{n} d} \cdot \frac{(d-1)^{n+2}}{d-2 m} \leq\left(\frac{d-1}{m}\right)^{n} \cdot \frac{(d-1)^{2}}{d}
$$

and so

$$
2^{n} \leq\left\{\frac{m(\tilde{d}-1)}{d-1}\right\}^{n}<\frac{(d-1)^{2}}{d}<d-1 \leq n
$$

which is a contradiction.

Proof of (II) and (III). Suppose $d<2 \tilde{d}$. From (I), we have $d>2 m$ and

$$
(\tilde{d}-1)^{n}<\frac{(d-1)^{n+2}}{m^{n} d} \leq \frac{(2 \tilde{d}-2)^{n}}{m^{n}} \cdot \frac{(d-1)^{2}}{d}
$$

hence $\left(\frac{m}{2}\right)^{n} \leq \frac{(d-1)^{2}}{d}<d-1 \leq n$, which shows $m \leq 2$.

Furthermore, if $d<2 \tilde{d}-1$ and $m=2$ (hence $d>4$ ), then

$$
\left(\frac{d}{2}\right)^{n} \leq(\tilde{d}-1)^{n}<\frac{1}{2^{n} d} \cdot \frac{(d-1)^{n+2}}{d-4} .
$$

Hence for $d \geq 7$,

$$
\left(\frac{d}{d-1}\right)^{n-5}<\frac{(d-1)^{7}}{d^{6}(d-4)}<1
$$


and this is a contradiction. Also for $d=5$ or 6 , the second inequality of (3.2) with $\tilde{d} \geq 5$ yields contradiction. Therefore, $m=1$ for $d<2 \tilde{d}-1$. Since $\operatorname{deg}(f)=m^{n} d / \tilde{d}<$ $2, f$ is an isomorphism, which shows (II).

For $d=2 \tilde{d}-1$, it is shown that $m \leq 2$. But if $m=1$ then $f$ is an isomorphism as before, which is impossible. This proves (III).

Next we treat the case $\tilde{d}=4$. The computation is basically the same, but it requires some care.

LEMma 3.2. Under the situation (*), assume $\tilde{d}=4$. Then (I) $2 m<d$ and

(II) if $d<2 \tilde{d}=8$, then $f$ is an isomorphism (hence $d=4$ ), except the possibility $(\tilde{d}=4, d=7$ and $m=2)$.

Proof. (i) First suppose $\frac{4}{3} m \geq d$. Then from (2.5) and (3.1),

$$
3^{n}<\frac{1}{m^{n} d} \cdot \frac{d(2 m-1)^{n+2}}{2 m(2 m-d)}<\frac{1}{m^{n}} \cdot \frac{(2 m)^{n+2}}{2 m \cdot \frac{2}{3} m}=3 \cdot 2^{n}
$$

which is impossible.

(ii) Next if $\frac{4}{3} m<d<2 m$, then

$$
3^{n}<\frac{1}{m^{n} d} \cdot \frac{d(2 m-1)^{n+2}}{2 m}<m \cdot 2^{n+1}<\frac{3}{4} d \cdot 2^{n+1} \leq 3(n+1) \cdot 2^{n-1} .
$$

This is impossible for $n \geq 6$. Now for $n<6$, we have $d \leq 6$ from Fano condition. Hence there are 4 possible cases within our degree bounds:

$(m=2, d=3),(m=3, d=5),(m=4, d=6)$ and $(m=4, d=7)$.

But all these yield contradictions to the first inequality of (3.3).

(iii) If $d=2 m$, then

$$
3^{n}<\frac{1}{m^{n} d} \cdot(n+2)(d-1)^{n+1}<\frac{(n+2) \cdot(2 m)^{n+1}}{2 m^{n+1}}=(n+2) \cdot 2^{n}
$$

which is impossible for $n \geq 5$. For $n<5$, there is only one possibility: ( $m=2, d=4)$. But this also yields a contradiction to the first inequality of (3.4).

From (i), (ii) and (iii), we get $2 m<d$, which shows (I).

Now to prove (II), assume $d<2 \tilde{d}=8$. Since $2 m<d$, we get $m \leq 3$. But if $m=3$, then $d=7$ and $\operatorname{deg}(f)=m^{n} d / \tilde{d}$ is a non-integer, which is absurd.

If $m=2$, then there are three possibilities: $d=5,6$ or 7 . But the former two cases $(d=5$ or 6$)$ yield contradictions to the inequality

$$
3^{n}<\frac{1}{m^{n} d} \cdot \frac{(d-1)^{n+2}}{d-2 m} .
$$

Hence we conclude $m=1$, except for the possibility $(m=2, d=7)$. Since $\operatorname{deg}(f)=$ $m^{n} d / \tilde{d}<2, f$ should be an isomorphism.

Now for $\tilde{d}=3$, we prove the following

LemMA 3.3. Under the situation (*), assume $\tilde{d}=3$. Then

(I) $m<3 d$ and

(II) if $d<2 \tilde{d}=6$, then $f$ is an isomorphism, except the possibility $(n=4, d=5, \tilde{d}=3$ and $m=3$ ). 
Proof. To show (I), suppose $d \leq \frac{1}{3} m$. Then from (2.1), (2.3) and (2.5), we get

$$
\frac{1}{3} \cdot \frac{2 \cdot 2^{n+2}-3+(-1)^{n}}{2}<\frac{1}{m^{n} d} \cdot \frac{d(2 m-1)^{n+2}}{2 m(2 m-d)}<\frac{1}{m^{n}} \cdot \frac{(2 m)^{n+2}}{2 m \cdot \frac{5}{3} m}
$$

Hence $\frac{1}{3}\left(2^{n+2}-2\right)<\frac{3}{10} \cdot 2^{n+2}$, which is a contradiction.

Now to show (II), assume $d<2 \tilde{d}=6$.

(i) $d=3$ : If $m \geq 2$, then $d<2 m$ and

$$
2^{n}<\frac{1}{m^{n} d} \cdot \frac{d(2 m-1)^{n+2}}{2 m(2 m-3)} .
$$

Hence

$$
\left(\frac{2 m}{2 m-1}\right)^{n-3}<\frac{(2 m-1)^{2}}{2 m(2 m-3)} \cdot\left(\frac{2 m-1}{2 m}\right)^{3}<1,
$$

which is a contradiction. Hence $m=1$ and $f$ is an isomorphism as before.

(ii) $d=4$ : Since $\operatorname{deg}(f)=m^{n} d / \tilde{d}$ is an integer, $m$ is a multiple of 3 and $2 m>d$. Now we have

$$
\frac{1}{\tilde{d}} c_{n}\left(\Omega_{\widetilde{X}}^{1}(2)\right) \geq \frac{2^{n+2}-2}{3} \geq \frac{5}{4} \cdot 2^{n} .
$$

Hence from (2.1) and (2.5),

$$
\frac{5}{4} \cdot 2^{n}<\frac{1}{m^{n}} \cdot \frac{(2 m-1)^{n+2}}{2 m(2 m-4)}
$$

and

$$
\left(\frac{2 m}{2 m-1}\right)^{n-3}<\frac{4}{5} \cdot \frac{(2 m-1)^{2}}{2 m(2 m-4)} \cdot\left(\frac{2 m-1}{2 m}\right)^{3}<1
$$

which is a contradiction. This proves $d \neq 4$.

(iii) $d=5$ : As before $m$ is a multiple of 3 and $2 m>d$. Now we have

$$
\frac{5}{4} \cdot 2^{n}<\frac{1}{m^{n}} \cdot \frac{(2 m-1)^{n+2}}{2 m(2 m-5)}
$$

and

$$
\left(\frac{2 m}{2 m-1}\right)^{n-3}<\frac{4}{5} \cdot \frac{(2 m-1)^{2}}{2 m(2 m-5)} \cdot\left(\frac{2 m-3}{2 m}\right)^{3}<1,
$$

for $m \geq 6$ and thus $m=3$.

Putting $m=3$ in (3.5), we have $(6 / 5)^{n}<10 / 3$, hence $n \leq 6$. It can be checked that the remaining cases $(\tilde{d}=3, d=5, m=3$ and $5 \leq n \leq 6)$ are also impossible by using the full inequality (2.1), (2.2) and (2.3). Therefore $d \neq 5$. Note that this line of arguments do not work for $(\tilde{d}=3, d=5, m=3$ and $n=4)$.

Proof of Main Theorem. By (I) of Lemma 3.1, 3.2 and 3.3, $\operatorname{deg}(f)=m^{n} d / \tilde{d}$ is bounded by some number which is determined by $d$ and $n$. By (II), (III) of Lemma 3.1 and (II) of Lemma 3.2 and 3.3, if $d<2 \tilde{d}-1$ then $f$ is an isomorphism. Also it is 
shown that if $d=2 \tilde{d}-1$, then $m=2$. The following lemma rules out this possibility and the Main Theorem is proved.

LEMMA 3.4. Let $X$ and $\tilde{X}$ be smooth hypersurfaces in $\mathbb{P}^{n+1}, n \geq 3$, of degree $d$, $\tilde{d}$ respectively, where $\tilde{d} \geq 2$. Let $f: X \rightarrow \widetilde{X}$ be a finite morphism and $m$ the number satisfying $f^{*} H_{\tilde{X}}=m H_{X}$. Then $m \tilde{d}-d \neq 1$.

Proof. (This is a repetition of C. Schuhmann's argument in [Sch], which concerned about the morphisms from cubic hypersurface to the quadric with $m=2$.)

Suppose there is a morphism $f$ with $m \tilde{d}-d=1$. In homogeneous coordinates, $f$ can be written as

$$
f=\left(\phi_{0}\left(x_{0}, \cdots, x_{n+1}\right), \cdots, \phi_{n+1}\left(x_{0}, \cdots, x_{n+1}\right)\right),
$$

where $\phi_{i}$ 's are homogeneous polynomials of degree $m$. Let $F$ (resp. $\tilde{F}$ ) be the homogeneous polynomial of degree $d$ (resp. $\tilde{d}$ ) defining $X$ (resp. $\tilde{X})$ in $\mathbb{P}^{n+1}$. Since $\tilde{F} \circ f=0$ on $X$, we have either $\tilde{F}\left(\phi_{0}, \cdots, \phi_{n+1}\right)=0$ or $\tilde{F}\left(\phi_{0}, \cdots, \phi_{n+1}\right)=F L$, where $L$ is a linear polynomial.

If $\tilde{F}\left(\phi_{0}, \cdots, \phi_{n+1}\right)=0$, then by restricting to a generic hyperplane, we have a nonconstant morphism $\left(\phi_{0}, \cdots, \phi_{n+1}\right): \mathbb{P}^{n} \rightarrow \widetilde{X}$. This contradicts Lazarsfeld's theorem (Theorem 1.1).

Hence we have $\tilde{F}\left(\phi_{0}, \cdots, \phi_{n+1}\right)=F L$. Let $H$ be the hyperplane defined by $L$. We claim that $\phi_{0}, \cdots, \phi_{n+1}$ have no common zeroes on $H$. This is obvious for $p \in X \cap H$. Now suppose $p \in H \backslash X \cap H$ and $\phi_{i}(p)=0$, for each $i$. Then

$$
0=\frac{\partial \tilde{F}\left(\phi_{0}, \cdots, \phi_{n+1}\right)}{\partial x_{j}}(p)=\frac{\partial(F L)}{\partial x_{j}}(p)=F(p) \frac{\partial L}{\partial x_{j}}(p) \text {. }
$$

Since $F(p) \neq 0, \frac{\partial L}{\partial x_{j}}(p)=0$ for each $j$, which is a contradiction.

Therefore $\phi_{0}, \cdots, \phi_{n+1}$ have no common zeroes on $H$ and $\left(\phi_{0}, \cdots, \phi_{n+1}\right)$ defines a morphism $H \rightarrow \widetilde{X}$. It is a nonconstant morphism since its restriction on $H \cap X$ is just $\left.f\right|_{H \cap X}$. This again contradicts Lazarsfeld's theorem.

Added in Proof. After this paper was written, D. Sheppard announced his results for the same problem in his thesis (see [Sh1], [Sh2]). Basically he used the same method as ours, but he also considered the case where the source hypersurface is allowed to be singular. Consequently, he could get a complete picture about morphisms between projective hypersurfaces where the target one is of general type. Also, he gave a different argument to prove that every morphism from a quintic hypersurface to a cubic hypersurface in $\mathbb{P}^{4}$ is constant.

Acknowledgement. I thank to Prof. Jun-Muk Hwang for his encouragement during the research. I also thank to the referee for careful reading and pointing out the exceptional case in Main Theorem.

\section{REFERENCES}

[A] E. AMERIK, On a problem of Noether-Lefschetz type, Compositio Mathematica, 112 (1998), pp. 255-271.

[ARV] E.AMERIK, M. ROVINSKY, AND A. VAN DE VEN, A boundedness theorem for morphisms between threefolds, Ann. Inst. Fourier (Grenoble), 49 (1999), pp. 405-415. 
[B] A. BEAUVILle, Endomorphisms of hypersurfaces and other manifolds, Internat. Math. Res. Notices, 2001:1 (2001), pp. 53-58.

[CS] K. CHO AND E. SATO, Smooth projective varieties dominated by smooth quadric hypersurfaces in any characteristic, Math. Z., 217:4 (1994), pp. 553-565.

[La] R. LAZARSFELD, Some applications of the theory of positive vector bundles, Springer Lecture Notes, 1092 (1984), pp. 29-61.

[Le] J. D. LEwIS, A survey of the Hodge conjecture, American Mathematical Society, Providence, RI, (1999).

[PS] K. H. PARANJAPE AND V. SRInivas, Self-maps of homogeneous spaces, Invent. Math., 98 (1989), pp. 425-444.

[P] T. PEternell, Manifolds of semi-positive curvature, Lecture Notes in Math., 1646 (1996), pp. 98-142.

[Sch] C. SchummanN, Mapping threefolds onto three-dimensional quadrics, Math. Ann., 306 (1996), pp. 571-581.

[Sh1] D. SHEPPARD, Towards characterizing morphisms between high dimensional hypersurfaces, arXiv: mathAG/032005.

[Sh2] D. SHEPPARD, Morphisms from quintic threefolds to cubic threefolds are constant, arXiv: mathAG/032006. 